\title{
Dynamical performance for science-mode stationkeeping with an external occulter
}

\author{
Sirbu, Dan; Karsten, Christian Vad; Kasdin, N Jeremy
}

Published in:

Proceedings of SPIE - The International Society for Optical Engineering

Link to article, DOI:

$10.1117 / 12.856361$

Publication date:

2010

Document Version

Publisher's PDF, also known as Version of record

Link back to DTU Orbit

Citation (APA):

Sirbu, D., Karsten, C. V., \& Kasdin, N. J. (2010). Dynamical performance for science-mode stationkeeping with an external occulter. Proceedings of SPIE - The International Society for Optical Engineering, 7731. https://doi.org/10.1117/12.856361

\section{General rights}

Copyright and moral rights for the publications made accessible in the public portal are retained by the authors and/or other copyright owners and it is a condition of accessing publications that users recognise and abide by the legal requirements associated with these rights.

- Users may download and print one copy of any publication from the public portal for the purpose of private study or research.

- You may not further distribute the material or use it for any profit-making activity or commercial gain

- You may freely distribute the URL identifying the publication in the public portal 


\title{
Dynamical performance for science-mode stationkeeping with an external occulter
}

\author{
Dan Sirbu ${ }^{1}$, Christian Vad Karsten ${ }^{1,2}$, and N. Jeremy Kasdin ${ }^{1}$ \\ ${ }^{1}$ Department of Mechanical and Aerospace Engineering, Princeton University \\ ${ }^{2}$ DTU Space, Technical University of Denmark
}

\begin{abstract}
An external occulter flown in precise formation with a telescope is being considered for high-contrast direct imaging of exoplanets as a viable mission scenario. In this paper, the dynamics about the Sun-Earth L2 region for an occulter-telescope constellation are considered in conjunction with fourth-body and solar radiation pressure acting as disturbing forces. An optimal observation window is defined in terms of both thrust required and the Sun-constellation geometry. By simulation, the effects of the stellar latitude and distance, the spacecraft separation, the magnitude of the disturbing forces, and on-off versus continuous thrusting are quantified on the thrusting profile needed to maintain precise alignment.
\end{abstract}

Keywords: Stationkeeping, External Occulter, High Contrast Imaging, Circularly Restricted Three Body Problem

\section{INTRODUCTION}

Since the 1992 discovery of the first extrasolar planet via pulsar timing, ${ }^{1}$ many new discoveries followed and quickly a new field of interest developed. To date, more than 450 extrasolar planets have been confirmed, but none have been characterized as terrestrial and most have been found by indirect methods, principally by radialvelocity measurements of the stellar wobble under the gravitational effect of the exoplanet. For the search and characterization of Earth-like exoplanets, a direct imaging method that is able to resolve a dim exoplanet in orbit to its much brighter host star has become a priority for the next decade.

Among the most promising methods for direct imaging is the use of external occulters, which are specially shaped opaque screens, that are flown in alignment with a telescope tens of thousands of kilometers along its line-of-sight to a target star. The occulter blocks out most of the light from a bright star before it reaches the telescope, only allowing light from the dim objects nearby the star to enter the telescope and thereby achieving the high-contrast needed for direct imaging. The special shape of the petals accounts for diffraction effects. Lyman Spitzer first ${ }^{2}$ proposed the idea of using occulters for finding extrasolar planets in 1962 and Simmons ${ }^{3,4}$ proposed using a shaped-pupil approach to replace an apodized occulter by a particular shape. Cash ${ }^{5}$ suggested a specific apodization function of the more general approach ${ }^{6}$ and replacing it by a starshaped occulter modeled on the shaped-pupil approaches. ${ }^{7}$ Vanderbei et al. ${ }^{8}$ developed optimal occulter apodizations that minimize the occulter distances and size while achieving the desired contrast over wide spectral bands.

This paper presents our validation of the full dynamic model of the occulter telescope constellation about the Sun-Earth L2 point. Through simulation the effects of certain design variables such as spacecraft separation, choice of target stars and thrusters are quantified. We use THEIA (Telescope for Habitable Exoplanets and Interstellar/Intergalactic Astronomy) as the reference mission profile. THEIA is a recently proposed externalocculter flagship-class mission. The current THEIA design calls for usage of a $4 \mathrm{~m}$ telescope and a single 40 $\mathrm{m}$ petal-shaped occulter operating at two different separations of 35,000 and 50,000 km corresponding to two wavelength bands to achieve the needed contrast to fully characterize a target planet. Recently a probe-class mission concept, dubbed $\mathrm{O}_{3}$ (Occulting Ozone Observatory), which uses a smaller telescope of $1.1 \mathrm{~m}$ and a 30 $\mathrm{m}$ occulter, has been proposed based on the same concepts as THEIA. From a stationkeeping perspective, both

Further author information: (Send correspondence to D.Sirbu)

D.Sirbu: E-mail: dsirbu@princeton.edu

Space Telescopes and Instrumentation 2010: Optical, Infrared, and Millimeter Wave,

edited by Jacobus M. Oschmann Jr., Mark C. Clampin, Howard A. MacEwen, Proc. of SPIE

Vol. 7731, 773152 - ㅇ 2010 SPIE · CCC code: 0277-786X/10/\$18 · doi: 10.1117/12.856361

Proc. of SPIE Vol. $7731773152-1$ 
missions are to be located at the Sun-Earth L2 point. For THEIA, the heavy telescope is to be placed in a ballistic halo orbit and the occulter is to be actively controlled for alignment while in science mode, while for $\mathrm{O}_{3}$ active control is performed on the lighter telescope which is then placed in alignment with the occulter now occupying the ballistic halo orbit trajectory.

The paper is organized as follows. Section 2 introduces the three-body dynamical model that governs the equations of motion of the spacecraft in the Sun-Earth dominated gravity field. Next, the kinematics of motion for the occulter-telescope constellation are considered in Section 3 and it is shown that the open-loop control law that maintains the desired alignment consists of a gravity gradient portion and a parallax correction term. The kinematics can be used to analytically generate the desired trajectory of the occulter and telescope constellation, as discussed in Section 4. In Section 5 disturbances are added to the model whereupon a brief technical discussion introduces mission-specifics for THEIA and $\mathrm{O}_{3}$ that are then used in simulations presented in Section 6. Lastly, Section 7 re-capitulates the main simulation results and trends in mission parameter choices before discussing future work.

\section{CIRCULARLY RESTRICTED THREE BODY MODEL}

The Sun-Earth L2 point is an unstable equilibrium point which provides several advantages for mission trajectory design, including relatively benign dynamical disturbances, low contamination from Earth's radiation, and good coverage from Earth for communication. Because of this, most of the occulter-class imaging missions that have been proposed (for example, THEIA, NWO, and $\mathrm{O}_{3}$ ) consider orbits about the L2 point. The well-known Circularly Restricted Three Body (CRTBP) model $^{9}$ is useful for describing the motion of a spacecraft around the L2 point. The assumptions are that the mass of the spacecraft is negligible compared to the two primaries (Earth and Sun in this case), thus not affecting their motion, and that the two primaries' motion is planar and circular. The details of the CRTBP model and the derivation of the relative equations of motion of the occulter with respect to the telescope are described elsewhere. ${ }^{10}$ For brevity only the equations of motion and basic definitions are reproduced below. First, an inertial frame is considered with its origin at the barycentre of the Sun and Earth primaries and which has two base unit vectors in the plane of the ecliptic and a third perpendicular and out of the ecliptic. The equations of motion for the telescope are considered in a corotating frame whose origin is also the barycentre, has a unit base vector pointing from the Sun to the Earth, a third unit vector outside of the ecliptic, and the remaining unit base is perpendicular to both. For numerical reasons it is best to use canonical units, wherein a canonical distance unit corresponds to $1 \mathrm{AU}$ and a canonical time unit is equal to the mean anomaly chosen such that mean angular motion of the primaries is unity in the rotating frame. $\mu$ is a massless parameter such that $\mu=\frac{\mu_{E}}{\mu_{H}+\mu_{E}}$ where $\mu_{E}$ is the combined Earth-Moon mass and $\mu_{H}$ is the Solar mass. Then the equations of motion for the telescope are given by:

$$
\begin{aligned}
& \ddot{x}_{T}=x_{T}+2 \dot{y}_{T}-\frac{1-\mu}{\left\|\mathbf{r}_{T / H}\right\|^{3}}\left(x_{T}+\mu\right)-\frac{\mu}{\left\|\mathbf{r}_{T / E}\right\|^{3}}\left(x_{T}+\mu-1\right)+w_{T x}+u_{T x} \\
& \ddot{y}_{T}=y_{T}-2 \dot{x}_{T}-\frac{1-\mu}{\left\|\mathbf{r}_{T / H}\right\|^{3}} y-\frac{\mu}{\left\|\mathbf{r}_{T / E}\right\|^{3}} y_{T}+w_{T y}+u_{T y} \\
& \ddot{z}_{T}=-\frac{1-\mu}{\left\|\mathbf{r}_{T / H}\right\|^{3}} z_{T}-\frac{\mu}{\left\|\mathbf{r}_{T / E}\right\|^{3}} z_{T}+w_{T z}+u_{T z}
\end{aligned}
$$

where $\mathbf{r}_{T}=\left[x_{T}, y_{T}, z_{T}\right]$ denotes the telescope position in the synodic frame, $\frac{{ }^{I} d \mathbf{r}_{T}}{d t}=\left[\dot{x}_{T}, \dot{y}_{T}, \dot{z}_{T}\right]$ is the inertial velocity of the telescope expressed as components in the synodic frame, $\mathbf{w}_{T}=\left[w_{T x}, w_{T y}, w_{T z}\right]$ the total disturbing acceleration acting on the telescope, and $\mathbf{u}_{T}=\left[u_{T x}, u_{T y}, u_{T z}\right]$ the total thrust acceleration acting on the telescope. The position from the Sun to the telescope is given by $\mathbf{r}_{T / H}$ and from the Earth to the telescope $\mathbf{r}_{T / E}$. The 
equations of motion for the occulter relative to the telescope are:

$$
\begin{aligned}
& \ddot{x}_{r}=x_{r}+2 \dot{y}_{r}+(1-\mu)\left(\frac{x_{T}+\mu}{\left\|\mathbf{r}_{T / H}\right\|^{3}}-\frac{x_{T}+x_{r}+\mu}{\left\|\mathbf{r}_{O / H}\right\|^{3}}\right)+\mu\left(\frac{x_{T}-1+\mu}{\left\|\mathbf{r}_{T / E}\right\|^{3}}-\frac{x_{T}+x_{r}-1+\mu}{\left\|\mathbf{r}_{O / E}\right\|^{3}}\right)+w_{r x}+u_{r x} \\
& \ddot{y}_{r}=y_{r}-2 \dot{x}_{r}+(1-\mu)\left(\frac{y_{T}}{\left\|\mathbf{r}_{T / H}\right\|^{3}}-\frac{y_{T}+y_{r}}{\left\|\mathbf{r}_{O / H}\right\|^{3}}\right)+\mu\left(\frac{y_{T}}{\left\|\mathbf{r}_{T / E}\right\|^{3}}-\frac{y_{T}+y_{r}}{\left\|\mathbf{r}_{O / E}\right\|^{3}}\right)+w_{r y}+u_{r y} \\
& \ddot{z}_{r}=(1-\mu)\left(\frac{z_{T}}{\left\|\mathbf{r}_{T / H}\right\|^{3}}-\frac{z_{T}+z_{r}}{\left\|\mathbf{r}_{O / H}\right\|^{3}}\right)+\mu\left(\frac{z_{T}}{\left\|\mathbf{r}_{T / E}\right\|^{3}}-\frac{z_{T}+z_{r}}{\left\|\mathbf{r}_{O / E}\right\|^{3}}\right)+w_{r z}+u_{r z}
\end{aligned}
$$

where $\mathbf{r}_{r}=\left[x_{r}, y_{r}, z_{r}\right]$ denotes the position of the occulter relative to the telescope, $\frac{{ }^{I} d \mathbf{r}_{r}}{d t}=\left[\dot{x}_{r}, \dot{y}_{r}, \dot{z}_{r}\right]$ the inertial velocity of the occulter relative position, $\mathbf{w}_{r}=\left[w_{r x}, w_{r y}, w_{r z}\right]$ the differential disturbing acceleration acting on the occulter with respect to the telescope, and $\mathbf{u}_{r}=\left[u_{r x}, u_{r y}, u_{r z}\right]$ the differential thrust acceleration acting on the occulter with respect to the telescope.

\section{OPEN-LOOP CONTROL}

In the following an open-loop control scheme based on the kinematics of the telescope and occulter will be introduced. Figure 1 is a simple planar schematic of the telescope and occulter relative to the star and barycenter. It is seen that the directional unit vector from the telescope to the star can be found from:

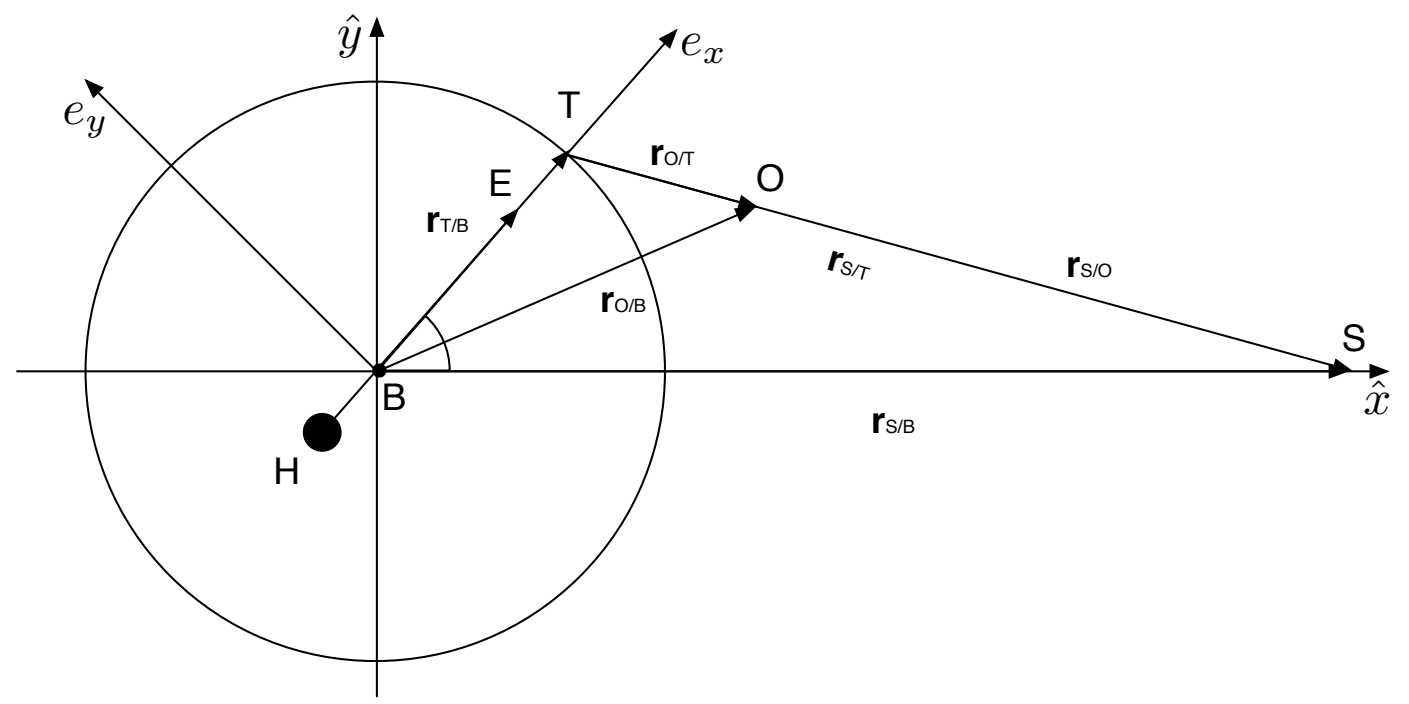

Figure 1. Schematic motion of the telescope (T) and Occulter (O) around the Sun(H)-Earth(E)-Moon Barycenter (B). The occulter is located a distance $\mathrm{R}$ from the telescope on the line-of-sight to the star (S)

$$
\begin{aligned}
\mathbf{r}_{T / B}+\mathbf{r}_{S / T} & =\mathbf{r}_{S / B} \\
\mathbf{r}_{S / T} & =\mathbf{r}_{S / B}-\mathbf{r}_{T / O} \\
\hat{\mathbf{r}}_{S / T} & =\frac{\mathbf{r}_{S / B}-\mathbf{r}_{T / O}}{\left\|\mathbf{r}_{S / B}-\mathbf{r}_{T / B}\right\|}
\end{aligned}
$$

It should be noted that $\mathbf{r}_{S / B}$ can be computed by conversion to ecliptic coordinates from equatorial coordinates provided by an astrometric catalogue (e.g., Hipparcos and Tycho). The occulter is located at a set separation $R$ from the telescope along the line-of-sight to the star:

$$
\mathbf{r}_{O / T}=R \hat{\mathbf{r}}_{S / T}
$$


The position of the occulter can then be expressed as:

$$
\mathbf{r}_{O / B}=\mathbf{r}_{T / B}+\mathbf{r}_{O / T}=\mathbf{r}_{T / B}+R \hat{\mathbf{r}}_{S / T}
$$

The velocity of the occulter in the inertial frame (I) is then given by:

$$
{ }^{\mathcal{I}} \mathbf{v}_{O / B}={ }^{\mathcal{I}} \mathbf{v}_{T / B}+R \frac{{ }^{\mathcal{I}} d \hat{\mathbf{r}}_{S / T}}{d t} .
$$

The acceleration of the occulter follows. From Newton's Second Law, the occulter's inertial acceleration is found from the total external force acting on the occulter due to the Sun and the Earth, $\mathbf{F}_{O}$, and the control acceleration $\mathbf{u}_{C}$ applied from the occulter's thrusters :

$$
{ }^{\mathcal{I}} \mathbf{a}_{O / B}={ }^{\mathcal{I}} \mathbf{a}_{T / B}+R \frac{{ }^{\mathcal{I}} d^{2} \hat{\mathbf{r}}_{S / T}}{d t^{2}}=\mathbf{F}_{O} / m_{O}+\mathbf{u}_{C} / m_{O} .
$$

Thus, the control acceleration $\mathbf{u}_{C}$ applied to the occulter needed to maintain the desired orbit can be found:

$$
\begin{aligned}
\mathbf{u}_{C} & =m_{O}\left(\mathbf{F}_{T} / m_{T}-\mathbf{F}_{O} / m_{O}+R \frac{{ }^{\mathcal{I}} d^{2} \hat{\mathbf{r}}_{S / T}}{d t^{2}}\right) \\
& =m_{O}(\underbrace{\left(\mu \frac{\mathbf{r}_{O / E}}{\left\|\mathbf{r}_{O / E}\right\|^{3}}+(1-\mu) \frac{\mathbf{r}_{O / H}}{\left\|\mathbf{r}_{O / H}\right\|^{3}}\right)}_{\mathbf{F}_{O} / m_{O}}-\underbrace{\left(\mu \frac{\mathbf{r}_{T / E}}{\left\|\mathbf{r}_{T / E}\right\|^{3}}+(1-\mu) \frac{\mathbf{r}_{T / H}}{\left\|\mathbf{r}_{T / H}\right\|^{3}}\right)}_{\mathbf{F}_{T} / m_{T}}+R \frac{{ }^{\mathcal{I}} d^{2} \hat{\mathbf{r}}_{S / T}}{d t^{2}}) .
\end{aligned}
$$

It can be seen from the above that alignment can be perfectly maintained by a control acceleration that is the summation of the gravity gradient given by the separation between the occulter and telescope $\mu \frac{\mathbf{r}_{T / E}}{\left\|\mathbf{r}_{T / E}\right\|^{3}}+(1-$ $\mu) \frac{\mathbf{r}_{T / H}}{\left\|\mathbf{r}_{T / H}\right\|^{3}}$ and a parallax correction term $R \frac{{ }^{\mathcal{I}} d^{2} \hat{\mathbf{r}}_{S / T}}{d t^{2}}$.

\section{TRAJECTORY GENERATION AND PARALLAX CORRECTION}

The desired trajectory of the occulter can be found analytically for all time depending on the position and velocity of the telescope. For the desired position of the occulter the previous formulation is invoked:

$$
\mathbf{r}_{O / B}=\mathbf{r}_{T / B}+R \hat{\mathbf{r}}_{S / T} .
$$

For the desired velocity of the occulter it holds that:

$$
{ }^{\mathcal{I}} \mathbf{v}_{O / B}={ }^{\mathcal{I}} \mathbf{v}_{T / B}+R \frac{{ }^{\mathcal{I}} d \hat{\mathbf{r}}_{S / T}}{d t}
$$

where the derivative term $\frac{I_{d \hat{\mathbf{r}}_{S / T}}}{d t}$ can be found analytically along each of its component directions:

$$
\begin{aligned}
& v x_{S / T}=\frac{\mathrm{vy}_{\mathrm{TB}}\left(x_{\mathrm{SB}}-x_{\mathrm{TB}}\right)\left(y_{\mathrm{SB}}-y_{\mathrm{TB}}\right)-\mathrm{vx}_{\mathrm{TB}}\left(\left(y_{\mathrm{SB}}-y_{\mathrm{TB}}\right)^{2}+\left(z_{\mathrm{SB}}-z_{\mathrm{TB}}\right)^{2}\right)+\mathrm{vZ}_{\mathrm{TB}}\left(x_{\mathrm{SB}}-x_{\mathrm{TB}}\right)\left(z_{\mathrm{SB}}-z_{\mathrm{TB}}\right)}{\left(\left(x_{\mathrm{SB}}-x_{\mathrm{TB}}\right)^{2}+\left(y_{\mathrm{SB}}-y_{\mathrm{TB}}\right)^{2}+\left(z_{\mathrm{SB}}-z_{\mathrm{TB}}\right)^{2}\right)^{3 / 2}} \\
& v y_{S / T}=\frac{\left(y_{\mathrm{SB}}-y_{\mathrm{TB}}\right)\left(\operatorname{vx}_{\mathrm{TB}}\left(x_{\mathrm{SB}}-x_{\mathrm{TB}}\right)+\mathrm{vZ}_{\mathrm{TB}}\left(z_{\mathrm{SB}}-z_{\mathrm{TB}}\right)\right)-\mathrm{vy}_{\mathrm{TB}}\left(\left(x_{\mathrm{SB}}-x_{\mathrm{TB}}\right)^{2}+\left(z_{\mathrm{SB}}-z_{\mathrm{TB}}\right)^{2}\right)}{\left(\left(x_{\mathrm{SB}}-x_{\mathrm{TB}}\right)^{2}+\left(y_{\mathrm{SB}}-y_{\mathrm{TB}}\right)^{2}+\left(z_{\mathrm{SB}}-z_{\mathrm{TB}}\right)^{2}\right)^{3 / 2}} \\
& v z_{S / T}=\frac{-\mathrm{vZ}_{\mathrm{TB}}\left(\left(x_{\mathrm{SB}}-x_{\mathrm{TB}}\right)^{2}+\left(y_{\mathrm{SB}}-y_{\mathrm{TB}}\right)^{2}\right)+\left(\mathrm{vx}_{\mathrm{TB}}\left(x_{\mathrm{SB}}-x_{\mathrm{TB}}\right)+\mathrm{vy}_{\mathrm{TB}}\left(y_{\mathrm{SB}}-y_{\mathrm{TB}}\right)\right)\left(z_{\mathrm{SB}}-z_{\mathrm{TB}}\right)}{\left(\left(x_{\mathrm{SB}}-x_{\mathrm{TB}}\right)^{2}+\left(y_{\mathrm{SB}}-y_{\mathrm{TB}}\right)^{2}+\left(z_{\mathrm{SB}}-z_{\mathrm{TB}}\right)^{2}\right)^{3 / 2}}
\end{aligned}
$$

This is a particularly useful result as it can be used to predict the insertion velocity of the occulter into sciencemode, and together with the position defines the reference orbit of the occulter. In a similar way an analytical formulation can be found for the parallax acceleration term, but nevertheless is negligibly small for all stars being considered compared to the other disturbances (we show this carefully in Figure 8). 


\section{DISTURBANCE MODEL}

The two primary disturbing forces in the L2 microgravity environment are the fourth-body effects of the Moon and the Solar radiation pressure. The effect of the Moon on the telescope and occulter is modeled as a periodic disturbing force that corresponds to the rotation of the Moon about the Earth assuming that is in plane and with a constant periodicity of approximately 27.3 days. This represents a slight simplification but agrees very well when a comparison is made to the force exerted by the Moon in a full four-body model:

$$
\begin{aligned}
& \mathbf{F}_{T / M}=-\mu \frac{\mu_{M}}{\mu_{E}} \frac{\mathbf{r}_{T / M}}{\left\|\mathbf{r}_{T / M}\right\|^{3}} m_{T} \\
& \mathbf{F}_{O / M}=-\mu \frac{\mu_{M}}{\mu_{E}} \frac{\mathbf{r}_{O / M}}{\left\|\mathbf{r}_{O / M}\right\|^{3}} m_{O} .
\end{aligned}
$$

Where in the above $\mu_{M}$ and $\mu_{E}$ represent the Moon mass and combined Earth-Moon mass respectively. Hence the differential acceleration due to Lunar disturbance on the occulter with respect to the telescope is:

$$
\mathbf{d}_{O}=\frac{\mathbf{F}_{T / M}}{m_{T}}-\frac{\mathbf{F}_{O / M}}{m_{O}}=-\mu \frac{\mu_{M}}{\mu_{E}}\left(\frac{\mathbf{r}_{T / M}}{\left\|\mathbf{r}_{T / M}\right\|^{3}}-\frac{\mathbf{r}_{O / M}}{\left\|\mathbf{r}_{O / M}\right\|^{3}}\right)
$$

where, for example for $\mathbf{r}_{T / M}=\mathbf{r}_{E / B}-\left[R_{M} \cos \left(\omega_{M} t\right) ; R_{M} \sin \left(\omega_{M} t\right) ; 0\right]$ and $\omega_{M}$ is the rotation frequency of the Moon about the Earth in a year.

The solar radiation pressure is modeled as a simplified disturbing force on the telescope and occulter respectively in accordance with McInner et al. ${ }^{11}$ For the telescope this disturbance is given by:

$$
\mathbf{w}_{T}=\frac{-\beta \mathbf{r}_{T / H}}{\left\|\mathbf{r}_{T / H}\right\|^{3}}
$$

where $\beta$ is a coefficient depending on the spacecraft coefficient of reflectivity, incident surface area, and fluctuations in the solar flux. Here it is assumed the coefficient is constant thus assuming no variations in surface area and solar flux from the initial point. As in the model for the MAXIM Pathfinder ${ }^{12}$ a coefficient of $\beta=5 \times 10^{-8}$ $\mathrm{m} / \mathrm{s}^{2}$ is assumed for the telescope. For simplicity, it is assumed that the occulter has a coefficient which is 40 times larger than that of the telescope based on a worst-case relative area differences and alignment to the Sun, hence $\beta_{O}=40 \beta_{T}$, and hence the relative disturbing effect on the occulter is $\mathbf{w}_{r}=\mathbf{w}_{O}-\mathbf{w}_{T}$

\section{SIMULATION AND RESULTS}

The THEIA occulter system is expected to have an approximate maximum mass of $6,000 \mathrm{~kg}$ and consists of a spacecraft and a $40 \mathrm{~m}$ occulter located at 35,000 or $50,000 \mathrm{~km}$ away. It accommodates a redundant system of NASA Evolutionary Xenon Thrusters (NEXT) and has solar power enough to fire two thrusters at a time with a maximum thrusting power of $6.9 \mathrm{~kW}$ each, a maximum thrust of $236 \mathrm{mN}$ and an ISP of 4190 . However, these thrusters are used primarily for slewing as during stationkeeping they produce a bright plume that could potentially contaminate observations. Hence, the occulter spacecraft is also equipped with a set of on/off hydrazine thrusters that must control transverse position to within $0.75 \mathrm{~m}$ during observations. ${ }^{13}$ During science-mode stationkeeping burn times a shutter is used to avoid light contamination from the plume. During observation periods the line-of-sight of the occulter and telescope is held on target and constrained to lie between 45 and 90 degrees from the Sun line. This is to avoid stellar leak or reflections off the occulter into the telescope. ${ }^{14}$

Figure 2 shows the results a simulation for THEIA with a 50,000 km separation over one year (roughly two periods of the Halo orbit) using a variable order Adams-Bashforth-Moulton integrator with 10 minute time steps. The right hand figure shows the total thrust required to maintain alignment for a selection of eight traget stars. All simulations started at the vernal equinox (March 21st) and the telescope follows a Halo orbit around L2 with synodic starting coordinates as in Sirbu et al. ${ }^{10}$ The halo orbit of the telescope is approximately stable over a year so no stationkeeping is applied on the telescope other than to correct the lunar and solar radiation disturbances. For the remainder of the paper, all simulations were performed using Epsilon Eridani as the target star. 

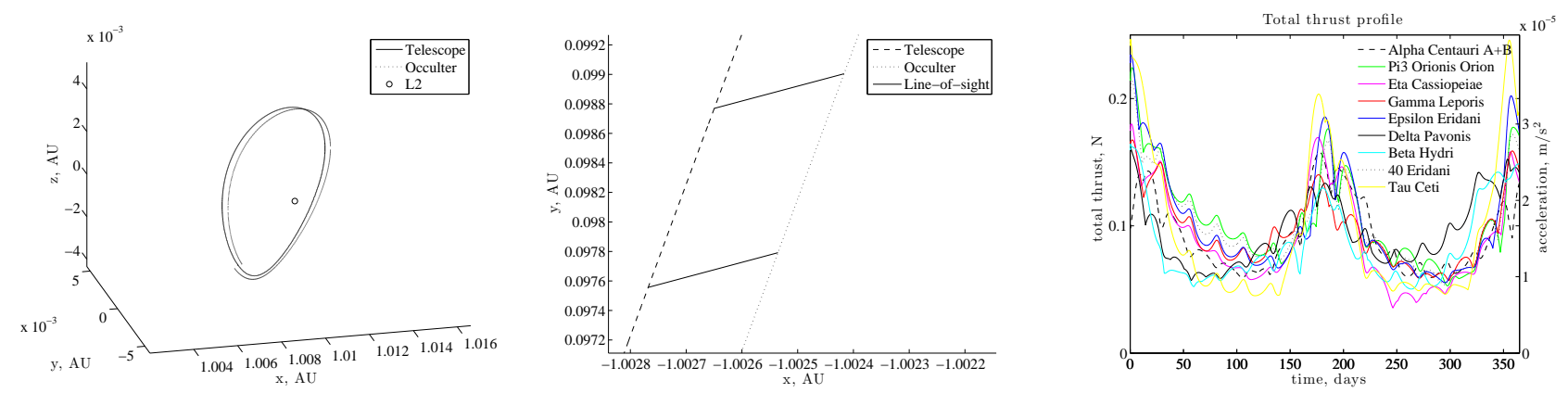

Figure 2. (left) Halo Orbit in the synodic frame over a period of 180 days. (middle) Line-of-sight from the telescope and occulter towards Epsilon Eridani in section of a halo orbit in the inertial frame. (right)Total thrust profile for different stars over the course of one year.
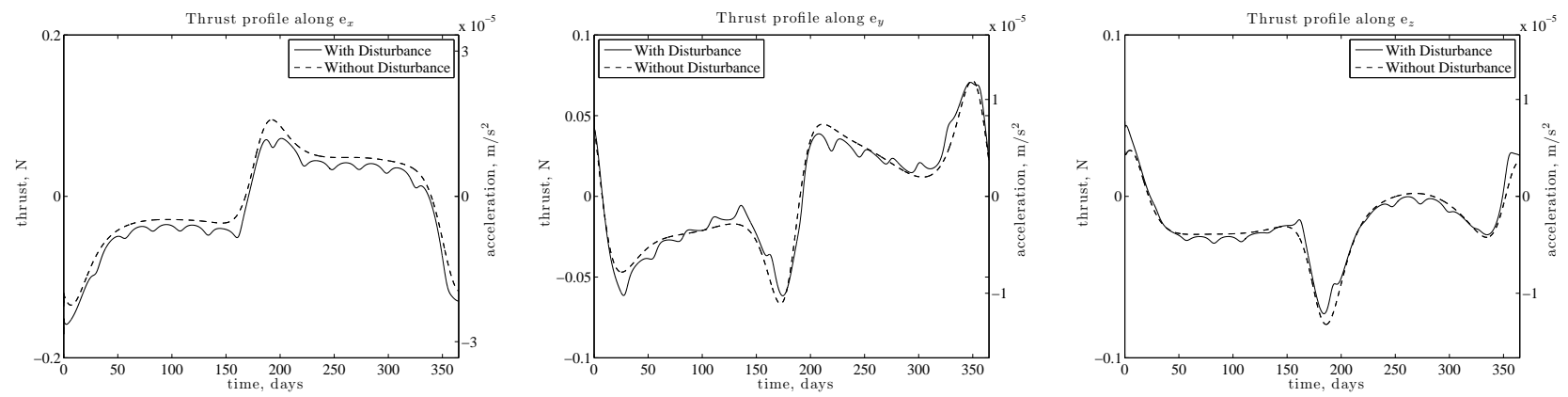

Figure 3. Effect of Lunar and Solar radiation pressure disturbances on the thrust profile along $e_{x}, e_{y}$, and $e_{z}$ (the three synodic components).

\subsection{Optimal Observation Window}

To determine an optimal observation window that minimizes thrust on the occulter to keep it aligned, the thrust profile is computed for a full year, and an 8-hour observation window around the point of lowest thrust is considered to be the optimal window. One other consideration is that the geometry of the telescope-occulter constellation with respect to the Sun must be such that the Sun does not glare on the telescope either directly or through reflections off the occulter. For example, for THEIA this means that the allowable angle $\theta$ between the vectors $\mathbf{r}_{O / T}$ and $\mathbf{r}_{H / T}$ must be constrained to be $45^{\circ} \leq \theta \leq 90^{\circ}$ such that it matches the sunshade installed on the telescope. Outside of this allowable region, a keep-out zone is defined; for $\theta>45^{\circ}$ the Sun is behind the occulter and in front of the telescope and so observations are impossible; for $90^{\circ}<\theta \leq 180^{\circ}$ the Sun is behind the telescope and its reflection off the occulter would likely cause significant glare.

\subsection{Effect of Disturbance}

The directional effect of the disturbances due to the Moon's gravitational field and the Solar radiation pressure is shown in Figure 3 and the total force and acceleration in Figure 4. The moon cycles are clearly seen and the disturbing forces have a significant effect on the overall thrusting level and cannot be ignored. However, the effects of Solar radiation are orders of magnitude smaller than the effect of the Moon and, hence, a simple model as proposed for Solar radiation pressure is appropriate.

\subsection{Effect of Separation Distance}

Ideally, the separation distance between the external occulter and the telescope would be increased to the maximum possible extent allowing for the inner working angle to be minimized and thus increase the observability of the habitable zone for faraway Solar Systems. However, this requirement must be balanced by the larger thrust needed for stationkeeping at large separation distances between the occulter and telescope. There are several 

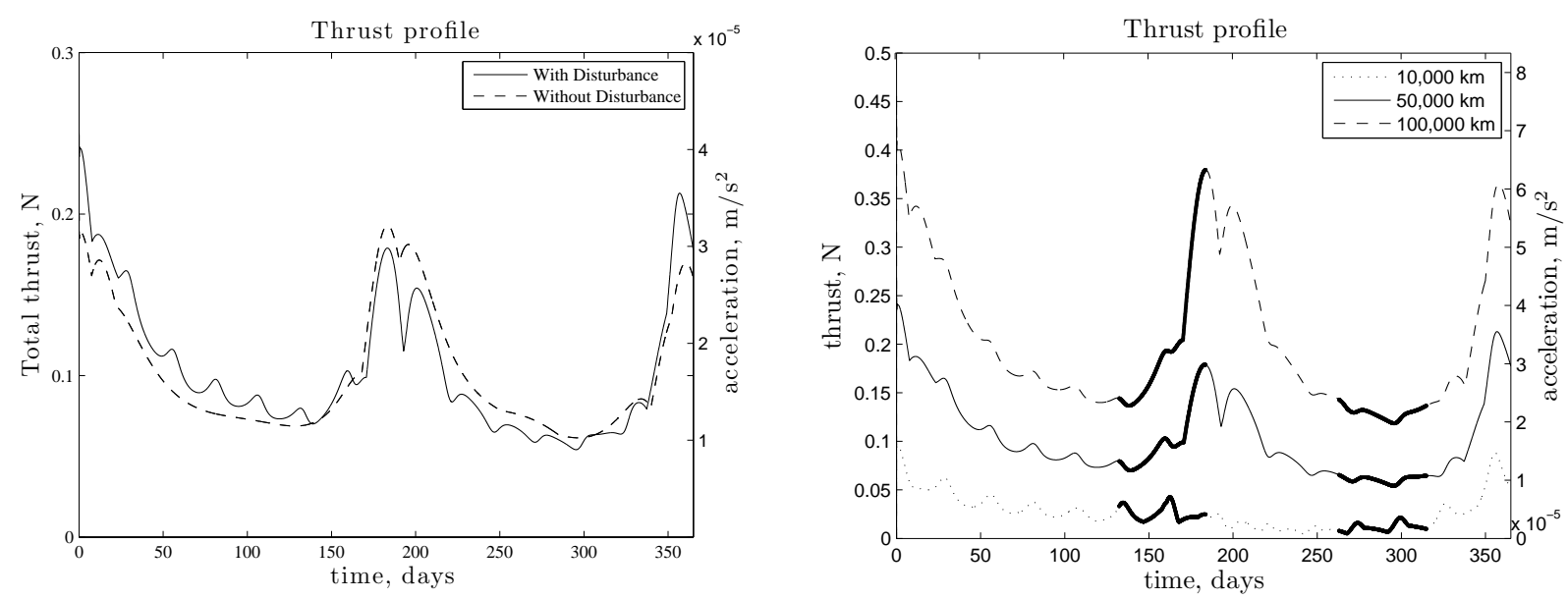

Figure 4. (left) Effect of disturbances on the total thrust profile. (right)Total thrust for three different separation distances of the occulter and telescope. Sections in bold outline correspond to observable periods when the Sun angle is favourabe
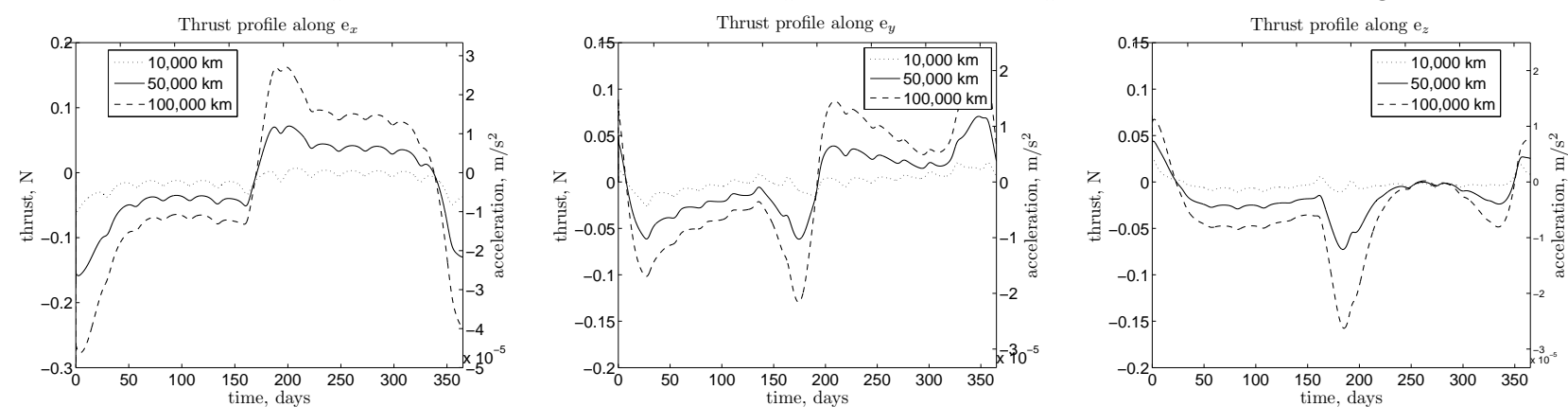

Figure 5. Thrust profile along $e_{x}, e_{y}$, and $e_{z}$ for different separation distances of the occulter and telescope constellation.

important considerations associated with increased thrust due to large separations. The thrusting required to maintain alignment must be within the physical limits of the onboard thrusters and the amount of onboard fuel. For continuous thrusters this requirement means that a stationkeeping window can be found over which the required thrusting profile is achievable (including a contingency). For on-off (chemical) thrusters, a realistic thrusting profile translates to more complicated considerations such as the number and length of cycles required to maintain the desired sub-metre alignment accuracy as well as the amount of fuel consumption. For a discussion of the cycle limitations associated with chemical thrusters, see Section 6.6. In the simulations shown in Figure 4 and Figure 5 the continuous-thrusting profile is considered for several separation distances, with 50,000 km as the separation proposed for THEIA. ${ }^{14}$ It is seen that the total thrust is proportional to the square of the separation distance as expected.

\subsection{Seasonal Effects}

Another effect upon the thrusting required to maintain the occulter in alignment with the telescope are seasonal effects, which in this case is the variation of the alignment halo orbit with respect to the inertial frame. The observable periods for the telescope-occulter system is dependent on the relative position of the Sun, target star, and position of the telescope on the Halo orbit. Hence over a mission lifetime less thrust intensive observation windows can be chosen since the period of a Halo orbit does not necessarily match with the revolution of the Earth. This effect is seen in Figure 6. For Epsilon Eridani this gives a ratio in total acceleration levels for stationkeeping during observations between worst and best windows of around 3 . 


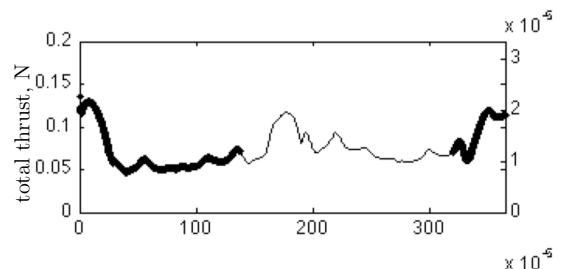

Seasonal Variations of Observation Windows
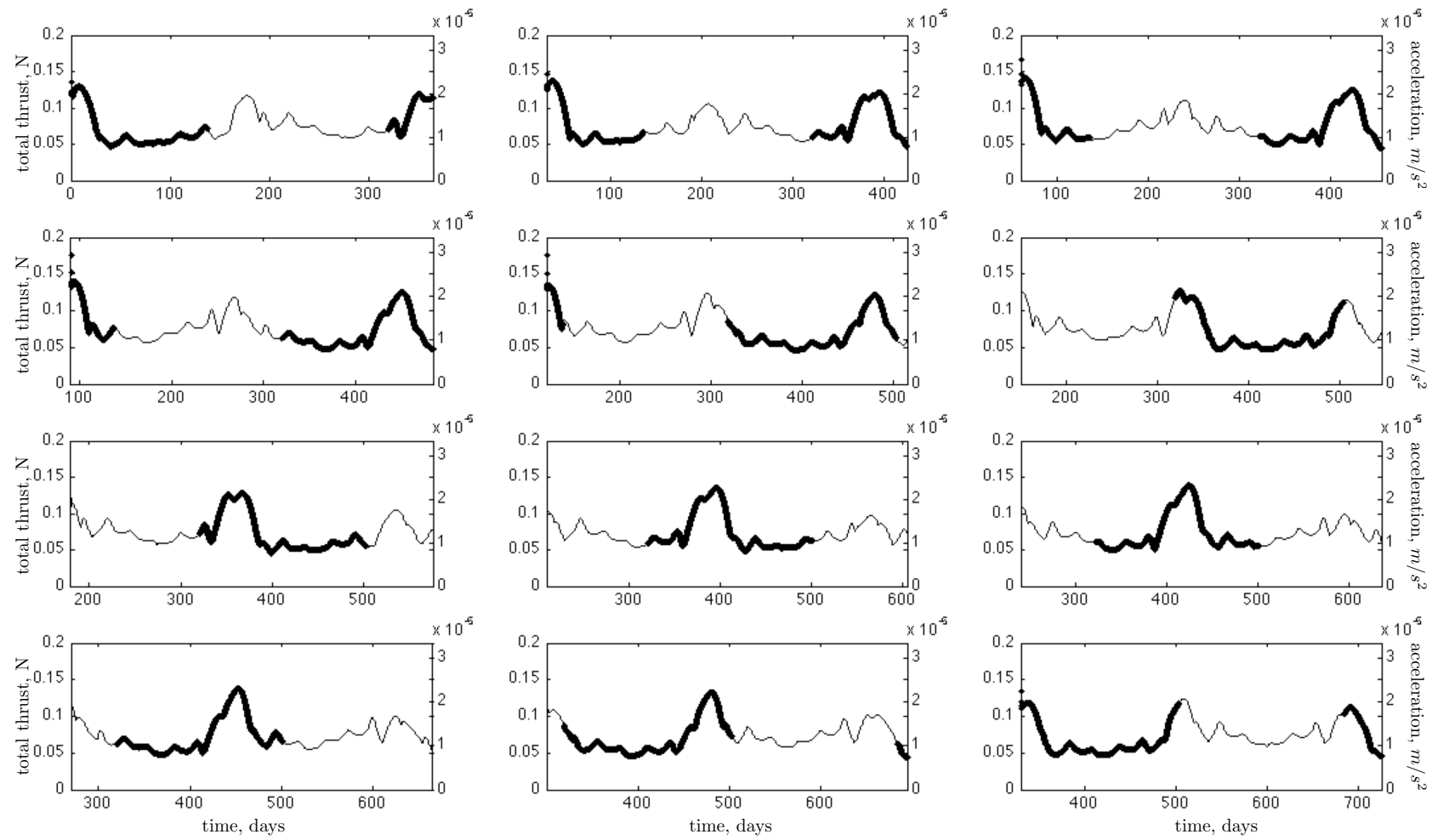

Figure 6. Total thrust profile for halo orbits starting at different times of the year. Upper left corner is March 21st and each increment is 30 days. The bolded sections correspond to observable periods due to favourable Sun angle.
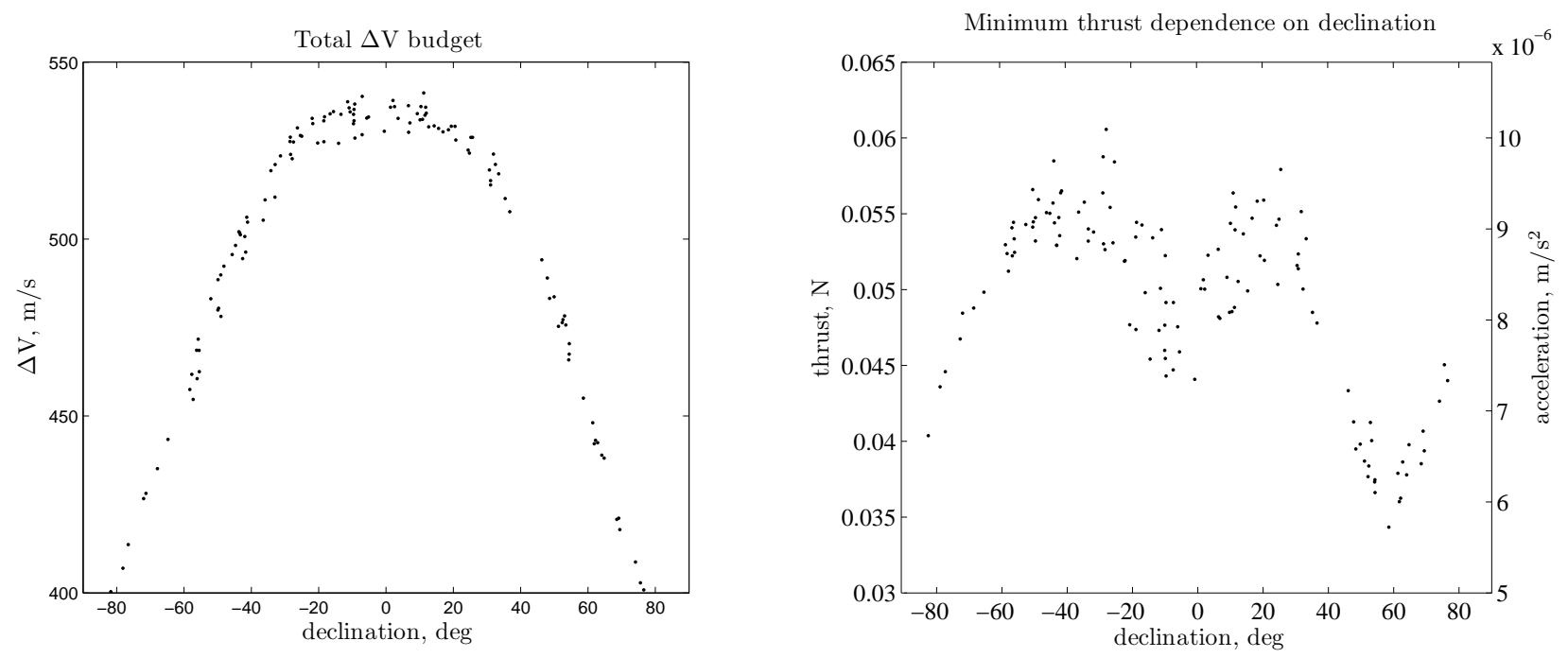

Figure 7. (left) The occulter is being kept in alignment with the telescope for an entire year and the reported stationkeeping budget is the average over five different starting times separated by 2 month increments. The results are summarized for all target stars and plotted as a function of stellar declination. (right) The optimal corresponding observation window for all target stars as a function of stellar declination is reported as an averaged minimum thrust. 

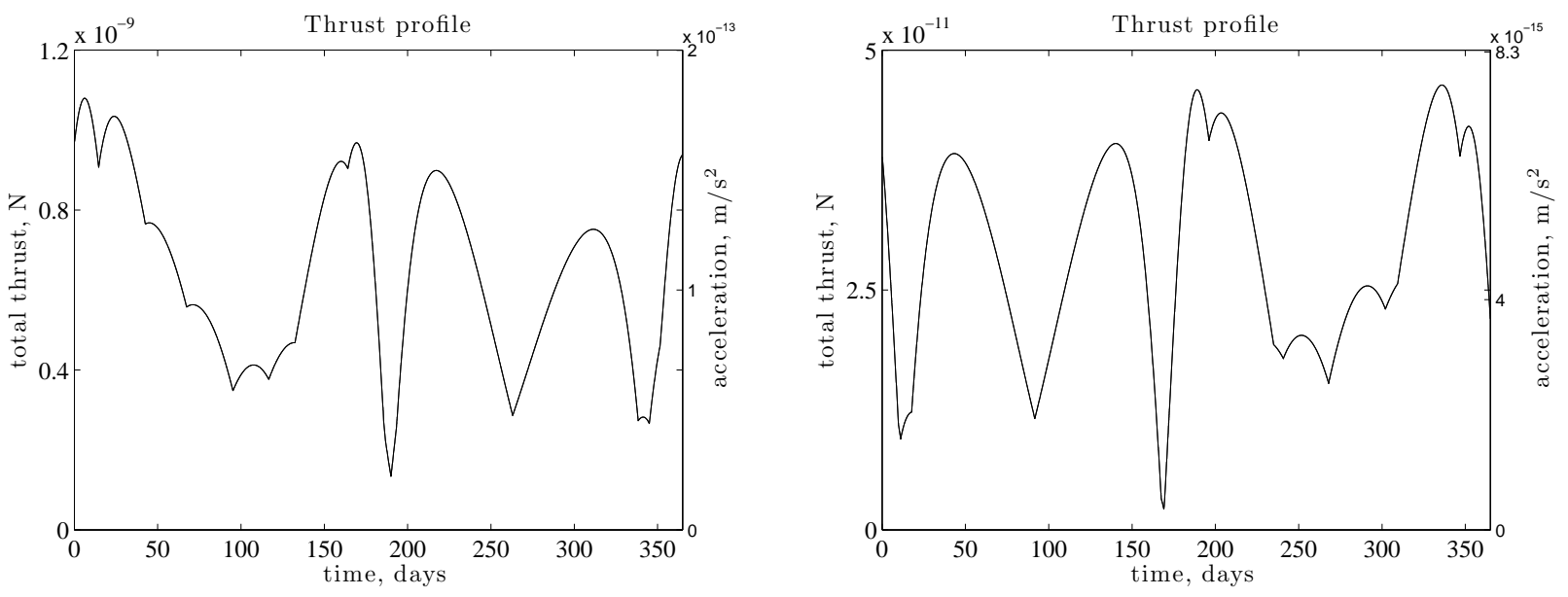

Figure 8. (left) Parallax correction thrust corresponding to HIP 71681, at a distance of 1.3 parsecs, and (right) HIP 30711, at a distance of 29.8 parsecs.

\subsection{Effect of Star Position}

The candidate star list for THEIA and $\mathrm{O}_{3}$ includes approximately 2300 stars within 30 parsecs from our Solar system. The target star list can be reduced to fewer than 131 stars by applying several filtering criteria including restricting the list to main sequence candidates, single stars (no binaries), observable habitable zone, and $\Delta$ mag $<$ 26 (for more details on selection of a dynamical target list see ${ }^{15}$ on DRM construction ). Of interest in this investigation is the effect of stellar declination on the occulter thrust profile and the corresponding observable windows of the star. The minimum thrust associated with the observable windows for different star declinations is shown in Figure 7; also plotted is the corresponding stationkeeping budget and whereas this shows an increase for stars nearer the ecliptic this is not necessarily matched by the occurrence of thrust minima. Different continuous thrust profiles are shown for 10 stars in Figure 2. The distance to the star only affects stationkeeping in the sense that for nearer stars the parallax correction is more significant; however, as shown in Figure 8, the thrust required to correct for parallax motion is negligible even for the closest stars in the target list.

\subsection{On/off Thrusters}

So far a continuous thrust profile has been considered for stationkeeping of the occulter; however the telescope imaging the target planet may be detrimentally affected by the always-on bright plumes produced by an ion thruster. To mitigate this effect for imaging, two solutions have been proposed: first, performing stationkeeping on the telescope (refer to the $\mathrm{O}_{3}$ discussion in Section 6.7) or using a bang-bang control scheme based on chemical thrusters that provide large thrusts over shorter durations. For this second approach, the science camera stops imaging while a burn is being applied on the occulter thrusters and is subsequently allowed to image while the occulter is drifting. To investigate the feasibility of a bang-bang approach, a thrusting profile is realized as illustrated in Figure 9 from the previously computed ideal continuous-thrusting profiles. The key thruster parameters are the thrust level and the burn time and, depending on the drift associated with the dynamical region, affect performance including maximum drift amount and time spent drifting. The length of each cycle is determined by the burn time and is bounded above by the maximum admissible drift which is a function of the lateral tolerance of the occulter alignment. ${ }^{13}$ Table 1 summarizes simulations using three different thrusters and the effect of the burn time during an eight-hour optimal observation window. It should be noted that a different thrust profile will arise for each different thrust direction but for the imaging condition listed above all burns must be matched together - thus the thrust profile for the highest drift component will dictate lengths of all cycles. 
Table 1. Summary of simulation results using chemical thrusting in the $e_{z}$ direction. For thrusters of strengths $1 \mathrm{~N}, 2 \mathrm{~N}$, and $5 \mathrm{~N}$, different burn times are chosen to quantify the drifting behaviour of the occulter. For lower burn times, more cycles are needed and this corresponds to a smaller overall drift while for shorter burn times cycles are more infrequent but a larger drift is present. This corresponds to a trade-off between lateral misalignment and an imaging cycle (time that science camera can be kept on).

\begin{tabular}{|c|c|c|c|c|}
\hline thrust & $\begin{array}{c}\text { time to } \\
\text { thrust }\left(t_{\mathrm{on}}\right) \\
(\mathrm{s})\end{array}$ & $\begin{array}{c}\text { drift } \\
\text { period }\left(t_{\text {off }}\right) \\
(\mathrm{s})\end{array}$ & $\begin{array}{c}\max \\
\text { drift } \\
(\mathrm{m})\end{array}$ & $\begin{array}{l}\text { \# cycles } \\
\text { in } 8 \text { hours }\end{array}$ \\
\hline \multirow{3}{*}{$1 \mathrm{~N}$} & 1 & 53.01 & 0.002 & 543 \\
\hline & 10 & 417.27 & 0.18 & 69 \\
\hline & 30 & 1492.14 & 1.76 & 19 \\
\hline \multirow{3}{*}{$2 \mathrm{~N}$} & 0.1 & 121.09 & 0.01 & 237 \\
\hline & 1 & 468.43 & 0.19 & 61 \\
\hline & 5 & 1359.77 & 1.13 & 21 \\
\hline \multirow{3}{*}{$5 \mathrm{~N}$} & 0.5 & 123.59 & 0.01 & 233 \\
\hline & 1 & 246.29 & 0.05 & 116 \\
\hline & 10 & 1232.35 & 1.27 & 23 \\
\hline
\end{tabular}
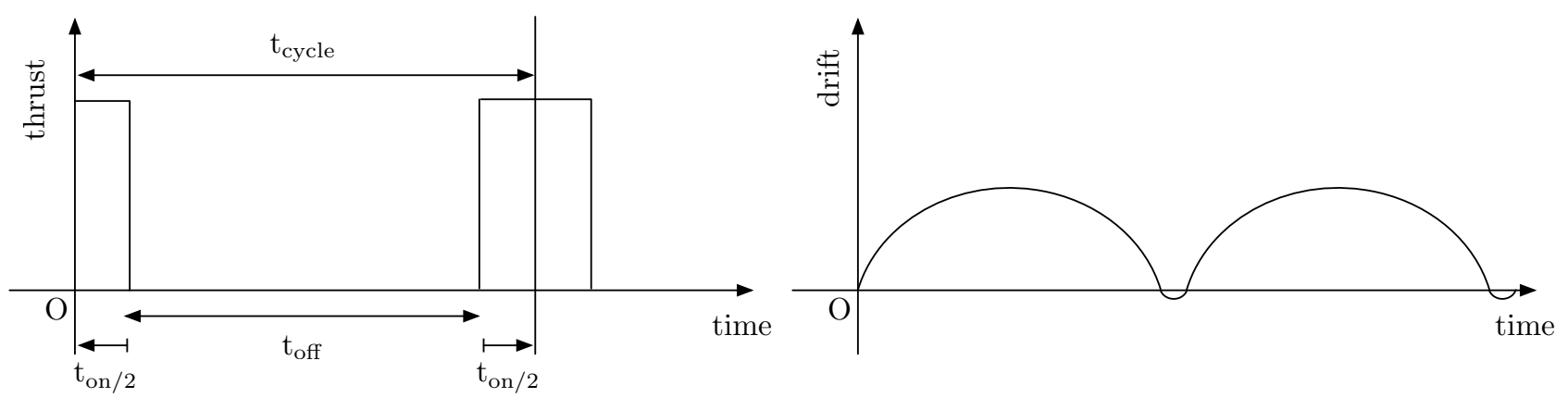

Figure 9. (left) Cycle schematic for the on/off thrusting profile. right) Corresponding drift for two on/off thrusting cycles after a burn is applied resulting in a maximum displacement against the drift and eventual return with the drift - longer drift periods correspond to longer periods when the camera can be turned on.

\subsection{Comparison of $\mathrm{O}_{3}$ and THEIA}

A major difference between THEIA and $\mathrm{O}_{3}$ is the constellation of the spacecraft. For THEIA the telescope is placed on a ballistic Halo orbit around L2 and the occulter is on the line-of-sight 50,000 km in the direction of the star. For $\mathrm{O}_{3}$ the setup is reversed and the occulter is kept at a Halo orbit around L2 with the telescope placed on the line-of-sight approximately 40,000 km in the direction away from the star. This means that stationkeeping will be done on the telescope rather than on the occulter during observations. The difference in accelerations can be seen in Figure 10 as well as the thrust, assuming a wet mass of the $\mathrm{O}_{3}$ telescope of $3,000 \mathrm{~kg}$ ( $\mathrm{see}^{16}$ for additional details) and a wet mass of the THEIA occulter of $6,000 \mathrm{~kg}$.

\section{CONCLUSION}

This paper presented a complete model and demonstrated the dynamical feasibility of a typical external occulter formation flying mission such as THEIA or $\mathrm{O}_{3}$. While assuming perfect control, the model can be used to study the thrust profiles for targets and conditions. In particular, we examined the variation in thrust over a year for changes in spacecraft separation, fourth-body and solar radiation pressure disturbing forces. The effects of using chemical thrusters on the occulter were also outlined and simulation results show the trade-off between 

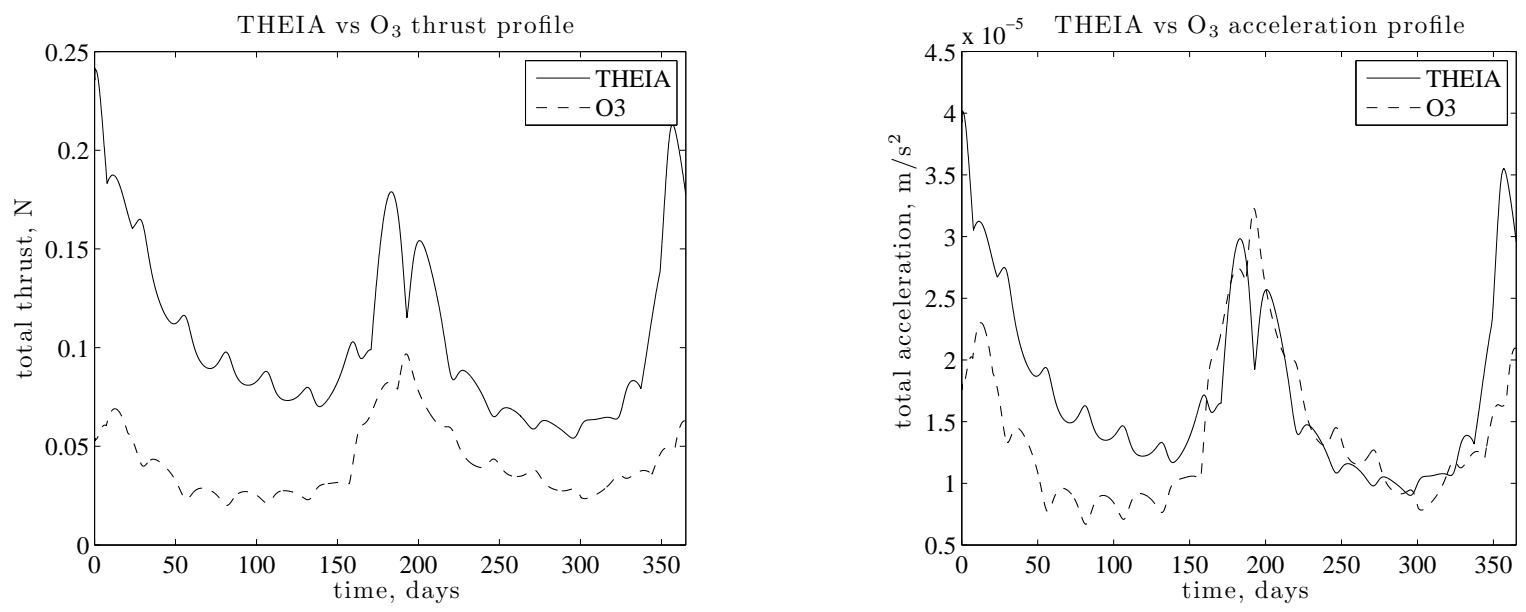

Figure 10. Comparison of thrust levels left) and acceleration levels (right) for THEIA and $\mathrm{O}_{3}$

increasing drift-time and minimizing lateral displacement. Lastly, the thrusting profiles for two different mission architectures, $\mathrm{O}_{3}$ and THEIA, were compared.

With a validated model in place, our next steps are to study realistic closed loop control algorithms. We plan to investigate both feedback only and feedforward controllers incorporating realistic navigational errors from the DSN, various time scales and bandwidths within the controller, and precise sensing models of the relative alignment. Various control architectures are being examined including optimal estimators to fuse the navigational and relative position information. A parallel laboratory effort at Princeton is validating the sensing scheme using a subscale version of the occulter system.

\section{ACKNOWLEDGMENTS}

The authors would like to acknowledge many helpful discussions with Dmitry Savransky who also provided code for determining the keep-out zone. DS acknowledges financial support from the Natural Sciences and Engineering Research Council of Canada and the Government of Alberta. CVK acknowledges financial support from Otto Mønsted Fonden and DTU Space.

\section{REFERENCES}

1. A. Wolszczan and D. A. Frail, "A planetary system around the millisecond pulsar psr1257 + 12," Nature 355, pp. 145-147, 1992.

2. L. Spitzer, "The beginnings and future of space astronomy.", American Scientist 50, pp. 473-484, 1962.

3. W. Simmons, N. Kasdin, R. Vanderbei, and W. Cash, "The new worlds observer: a mission for highresolution spectroscopy of extrasolar terrestrial planets.," Proceedings of SPIE Conference on Astronomical Telescopes and Instrumentation 5490, 2004.

4. W. Simmons, "A pinspeck camera for exo-planet spectroscopy.," Master's thesis, Princeton University, Dept. of Mechanical and Aerospace Engineering, 2005.

5. W. Cash, "Detection of earth-like planets around nearby stars using a petal-shaped occulter.," Nature 442, pp. 51-53, 2006.

6. C. Copi and G. Starkman, "Big occulting steerable satellite," Astrophysical Journal 532, pp. 581-592, 2000.

7. R. Vanderbei, D. Spergel, and N. Kasdin., "Circularly symmetric apodization via star-shaped masks.," Astrophysical Journal 599, pp. 686-694, 2003.

8. R. Vanderbei, E. Cady, and N. Kasdin, "Optimal occulter design for finding extrasolar planets.," Astrophysical Journal 665, pp. 794-798, 2007.

9. M. Valtonen and H. Kartunnen, Three-body problem, Cambridge University Press, 2006. 
10. D. Sirbu, E. J. Chen, M. S. Isakowitz, R. L. Johnson, D. W. Maas, and N. J. Kasdin, "Stationkeeping for an occulter-based exoplanetary imaging mission," 33rd Guidance $\mathcal{E}$ Control Conference, 2010.

11. C. McInner, J. Simmons, and E. MacDonald, "Solar sail parking in restricted three-body systems," Journal of Guidance, Control, and Dynamics 17, pp. 399-406, 1994.

12. P. Gurfil, M. Idan, and N. J. Kasdin, "Adaptive neural control of deep-space formation flying," Journal of Guidance, Control, and Dynamics 26(3), pp. 491-501, 2003.

13. P. J. Dumont, S. B. Shaklan, E. Cady, N. J. Kasdin, and R. Vanderbei, "Analysis of external occulters in presence of defects," Proc. of SPIE 7440, 2009.

14. N. J. Kasdin, "Theia - telescope for habitable exoplanets and intersteallar/intergalactic astronomy." White Paper Submitted to NRC ASTRO-2010 Survey.

15. D. Savransky and N. Kasdin, "Design reference mission construction for planet finders," Proc. of SPIE 7010, p. 200, 2008.

16. N. J. Kasdin, D. N. Spergel, P. D. Lisman, S. B. Shaklan, D. Savransky, E. Cady, E. L. Turner, R. Vanderbei, M. W. Thomson, S. R. Martin, K. Balasubramamian, S. H. Pravdo, Y. Fujii, and Y. Suto, "O $\mathrm{O}_{3}$ : The occulting ozone observatory." American Astronomical Society, Poster. 